\title{
Neural Networks Based Adaptive Consensus for a Class of Fractional-Order Uncertain Nonlinear Multiagent Systems
}

\author{
Jing Bai ${ }^{1}$ and Yongguang $\mathrm{Yu} \mathbb{(}^{2}$ \\ ${ }^{1}$ School of Mathematics and Physics, University of Science and Technology, Beijing 100083, China \\ ${ }^{2}$ Department of Mathematics, Beijing Jiaotong University, Beijing 100044, China \\ Correspondence should be addressed to Yongguang Yu; ygyu@bjtu.edu.cn
}

Received 10 September 2018; Revised 25 October 2018; Accepted 31 October 2018; Published 12 November 2018

Academic Editor: Mahdi Jalili

Copyright (c) 2018 Jing Bai and Yongguang Yu. This is an open access article distributed under the Creative Commons Attribution License, which permits unrestricted use, distribution, and reproduction in any medium, provided the original work is properly cited.

Due to the excellent approximation ability, the neural networks based control method is used to achieve adaptive consensus of the fractional-order uncertain nonlinear multiagent systems with external disturbance. The unknown nonlinear term and the external disturbance term in the systems are compensated by using the radial basis function neural networks method, a corresponding fractional-order adaption law is designed to approach the ideal neural network weight matrix of the unknown nonlinear terms, and a control law is designed eventually. According to the designed Lyapunov candidate function and the fractional theory, the systems stability is proved, and the adaptive consensus can be guaranteed by using the designed control law. Finally, two simulations are shown to illustrate the validity of the obtained results.

\section{Introduction}

Consensus of multiagent systems (MASs) in distributed coordination has been widely discussed in different fields, such as biological formation flocking, consensus of intelligent robots, distributed sensor networks, group decision making, and multiple collaborative manipulator [1]. Consensus means that the states of agents reach a common state through local neighbors information as time goes on. Consensus has become a hot topic, which has been researched from different perspectives based on the practical demands, for example, the consensus problem under different communication topologies: the fixed topology or switching topology $[2,3]$; the consensus problem under different dynamical structures, which include single-integrator dynamics or higher-integrator dynamics [4, 5]; the consensus problem with time delay: input delay or communication delay $[6,7]$; the consensus problem with unknown factors or external disturbance $[8,9]$; the leaderless consensus or leader-follower consensus [7, 10]; the group consensus problem [11]; and the design of control laws based on different control methods $[12,13]$.

The above results are based on the study of integer-order MASs, as we know that the integer-order systems cannot describe the complex materials or processes very well in some applications, for example, the viscous material, the food seeking of microbes, the collective motion of bacteria, and radial groundwater flow to or from a well [14]. In addition, it is difficult to describe the systems by the integer-order MASs when agents work in the complex environment, such as the viscous environment with micro-organisms, sand, and grass [15]. Fractional-order systems have great performance in the memory and hereditary $[16,17]$ and can provide an excellent tool to solve above problems. Therefore, it is meaningful to study the consensus problems by using fractional-order MASs.

In recent years, the consensus problem of fractional-order multiagent systems has been investigated widely. Based on different requirements, the researchers have mainly studied the leaderless consensus problem and the leader-follower consensus problem, where the single-integrator MASs and double-integrator MASs $[18,19]$ have been considered from various perspectives. For example, time delay can cause poor robust and instability of MASs; the authors in $[20,21]$ have studied the effectiveness of systems convergence when MASs have input delay or communication delay, in which, the systems stability and the maximal allowed time delay have 
been mainly discussed, and the fractional frequency domain analysis method and the generalized Nyquist criterion have been mainly used. In addition, various valid control laws are designed to solve different problems based on different control methods, such as the adaptive control method [22], the fractional-order PID control method [23], the sliding mode control method [24], and the event triggering control method [25]. Moreover, MASs have been studied from the linear systems to the nonlinear systems [26-31], where researchers have focused on studying the nonlinear fractional-order MASs; they can cause systems degraded performance or instability, and they are both inevitable in practical applications for the dynamic systems or the effectiveness of the external disturbance; therefore, it is necessary to consider the nonlinear MASs.

Until now, there are few results on the study of the fractional-order nonlinear MASs with external disturbance, and due to the complexity of the nonlinear fractional-order systems and the deficiency of corresponding theory, the researchers have usually assumed that the nonlinear term or the external disturbance term is known in MASs; at the same time, the former satisfies the Lipschitz condition, and the latter is bounded. But as we know, the above assumptions are restrictive. On the one hand, the nonlinear term and the external disturbance term cannot usually be observed, and it is not easy to design the corresponding control law to compensate for their effectiveness for the MASs. On the other hand, the assumptions about the Lipschitz condition and boundedness are difficult to be satisfied for the practical nonlinear MASs. Therefore, it is significant to design the valid control laws, which is not necessary to know the specific information on the nonlinear term and the external disturbance term in MASs. Meanwhile, the limitations for the nonlinear term and the external disturbance term can be reduced. Up to now, some results $[28,32]$ have designed the observer-type control law to avoid obtaining the unknown information in fractional-order MASs, but most of them discuss the linear MASs.

For the above unknown nonlinear MASs, the neural networks (NNs) based control methods can provide a powerful tool due to their universal approximation property and learning ability. The existing results have proved that NNs can approximate the nonlinear terms to any desired accuracy when the number of neurons is large enough [33]. However, for the most of above results, large adaptive parameters need to be estimated in the learning process when NNs are employed to approximate the unknown nonlinear terms, so that the learning time is unacceptably large; this drawback will increase the online computation burden greatly when the control laws are implemented. Radial Basis Function Neural Networks (RBFNNs) have been proposed with linear property to solve above problem, which only need to adjust a small number of parameters. In [34], by using RBFNNs, the authors have considered the tracking problem of uncertain nonlinear MASs, where small number parameters are updated; it alleviates the systems computation burden, and thus the running cost is reduced greatly. The existing results $[33,34]$ about NNs based control method focus on the study of integer-order MASs, and there are few results on the fractional-order uncertain nonlinear MASs; the tracking problem has been studied in [35], while the NNs based method is applied to estimate the uncertain nonlinear terms. Therefore, it is meaningful to solve consensus of fractionalorder nonlinear MASs by using the RBFNNs based control methods.

Motivated by the above discussion, in this paper we will apply the NNs based control method to solve the adaptive consensus problem for the fractional-order uncertain nonlinear MASs with external disturbance. At the beginning, to study consensus of fractional-order nonlinear MASs, we firstly assume that the nonlinear terms in systems are known and then design a valid control law based on the known nonlinear terms information. Secondly, based on the above study, the NNs based control law is designed to deal with the consensus problem when the nonlinear terms are unknown, the systems stability is proved based on matrix theory and the fractional-order stability theory. At last, two simulations are given to show the effectiveness of the obtained results. Compared to the existing results on fractionalorder MASs, this paper mainly has the following differences. Firstly, compared with the results about the fractional-order nonlinear MASs [27-31], this paper discusses the general fractional-order nonlinear MASs with external disturbance, where the Lipschitz condition and boundedness condition are not necessary for the nonlinear terms. Secondly, different from the existing control methods [22-25], the paper designs a developed RBFNNs based approximation control law to overcome the unknown nonlinear terms in fractional-order MASs. Finally, compared to the most of NNs based control methods $[33,35,36]$, the developed RBFNNs based control law only needs to update a small number of parameters for fractional-order MASs, which can reduce the systems computation burden effectively.

The rest of this paper is arranged as follows. In Section 2, the corresponding fractional calculus theory and graph theory are given, and we focus on introducing the RBFNNs method to approach the unknown nonlinear terms. In Section 3, the adaptive consensus with the unknown nonlinear terms is discussed based on RBFNNs approximation technique, a valid control law is designed and a fractionalorder adaption law is proposed to estimate the ideal RBFNNs weight matrix. In Section 4, two simulations are drawn to verify the obtained results. Finally, the paper summarization is concluded in Section 5.

\section{Preliminaries}

2.1. Fractional Calculus. In the study of fractional-order MASs, the definitions of fractional-order derivative play important roles, and different definitions have different advantages, where Caputo fractional-order derivative definition can describe the initial states of multiple agents very well; therefore, Caputo fractional derivative definition as follows will be applied throughout this paper [14]

$$
{ }_{a}^{C} D_{t}^{\alpha} x(t)=\frac{1}{\Gamma(m-\alpha)} \int_{a}^{t} \frac{x^{(m)}(\tau)}{(t-\tau)^{\alpha-m+1}} d \tau,
$$


where ${ }_{a}^{C} D_{t}^{\alpha}$ represents the Caputo derivative, $\alpha$ is an arbitrary real number, which describes the system order, $m$ is an integer constant, and it satisfies $m-1<\alpha<m$, and the Gamma function is given as $\Gamma(p)=\int_{0}^{+\infty} t^{p-1} e^{-t} d t$, which satisfies

$$
\Gamma(p+1)=p \Gamma(p)
$$

where $p$ is an arbitrary real number.

The Laplace transform of Caputo fractional derivative plays a key role, and it has the following form:

$$
L\left\{x^{(\alpha)}(t)\right\}=s^{\alpha} X(s)-\sum_{k=0}^{m-1} s^{\alpha-k-1} x^{(k)}(0),
$$

$X(s)=L\{x(t)\}=\int_{0}^{+\infty} e^{-s t} x(t) d t$ is defined.

The order $\alpha$ in any interval can be translated to consider the interval $\alpha \in(0,1)$; therefore, we just discuss the interval $\alpha \in(0,1)$, and then, the Laplace transform of Caputo fractional derivative can be written as the following form.

$$
L\left\{x^{(\alpha)}(t)\right\}=s^{\alpha} X(s)-s^{\alpha-1} x(0) . \quad \alpha \in(0,1)
$$

To analyze the systems stability, we need to introduce the following Mittag-Leffler function:

$$
E_{\alpha, \beta}(z)=\sum_{k=0}^{\infty} \frac{z^{k}}{\Gamma(k \alpha+\beta)}
$$

and, specially, if $\beta=1$ and $\alpha>0$, the Mittag-Leffler function becomes

$$
E_{\alpha}(z)=\sum_{k=0}^{\infty} \frac{z^{k}}{\Gamma(k \alpha+1)}
$$

Definition 1. It is Mittag-Leffler stable for the system stability if

$$
\|x(t)\| \leq\left[m\left(x\left(t_{0}\right)\right) E_{\alpha}\left(-\lambda\left(t-t_{0}\right)^{a}\right)\right]^{b},
$$

where $\|\cdot\|$ defines arbitrary norm, $\lambda>0, b>0, m(0)=0$, the locally Lipschitz condition for $m(x) \geq 0$ is satisfied on $x \in R^{n}$, and the Lipchitz constant is defined as $m_{0}$.

To analyze the system stability, we give the following inequality by using the result in [24].

Lemma 2. For any time instant $t \geq t_{0}$, the following inequality can be obtained if $x(t) \in R^{n}$ is a continuous and derivable function,

$$
\frac{1}{2}{ }_{t_{0}}^{C} D_{t}^{\alpha}\left(x^{T}(t) L x(t)\right) \leq x^{T}(t) L_{t_{0}}^{C} D_{t}^{\alpha} x(t)
$$

where $L$ is a symmetric positive semidefinite matrix and $\alpha \epsilon$ $(0,1)$.
2.2. Graph Theory. This paper considers the fixed undirected communication topology graph $G=(V, E, A)$, where $V=$ $\left\{v_{1}, v_{2}, \ldots, v_{n}\right\}$ is the set of nodes in the graph, $v_{i}$ represents the $i$ th follower, and $E \subseteq V \times V$ is the set of edges in the graph, and define $e_{i j}=\left(v_{i}, v_{j}\right)$. If $e_{i j} \in E, e_{j i}=\left(v_{j}, v_{i}\right) \in E$. The set of neighbors for the node $v_{i}$ in the graph is denoted as $N_{i}=\left\{v_{j} \mid\left(v_{j}, v_{i}\right) \in E\right\}$. We call that an undirected path is connected if every pair node has an undirected path, where a path is represented as $\left(v_{1}, v_{2}\right),\left(v_{2}, v_{3}\right), \ldots$, and $v_{i} \in V$.

The weighted adjacency matrix $A$ and the Laplace matrix $L$ can show the undirected graph $G$ very well, where $A=$ $\left[a_{i j}\right] \in R^{n \times n}$, and $a_{i j}=a_{j i}>0$ if $\left(v_{j}, v_{i}\right) \in E$, else $a_{i j}=0$. $L=\left[l_{i j}\right] \in R^{n \times n}$, and $l_{i i}=\sum_{j \in N_{i}} a_{i j}, l_{i j}=-a_{i j}, i \neq j$. The Laplace matrix $L$ satisfies the following important property.

$$
\begin{gathered}
l_{i j} \leq 0, \quad i \neq j, \\
\sum_{j=1}^{n} l_{i j}=0 . \quad i=1, \ldots, n
\end{gathered}
$$

In this paper, we consider the undirected graph, then the Laplace matrix $L$ is a symmetric positive semidefinite, and the Laplace matrix has at least one zero eigenvalue, whose corresponding eigenvector is $\mathbf{1}=(1,1, \ldots, 1)^{T}$.

The following lemma [37] can be guaranteed.

Lemma 3. The Laplace matrix $L$ is a symmetric matrix for the undirected connected graph $G=(V, E, A)$, and its eigenvalues have the following relationship:

$$
0=\lambda_{1}<\lambda_{2} \leq \cdots \leq \lambda_{n}
$$

where $\lambda_{2}$ is the minimal positive eigenvalue of $L$, which can analyze the rate of consensus convergence.

2.3. Radial Basis Function Neural Networks and Function Approximation. In this paper, the fractional-order uncertain nonlinear systems are mainly investigated; as a valid NNs based control method, the radial basis function neural networks (RBFNNs) based method will be applied to estimate the nonlinear terms in MASs; RBFNNs based method has excellent property in the function approximation and learning ability [38]. Consider the smooth nonlinear term $f(x): R^{n} \longrightarrow R^{m}$; it can be approximated by the following RBFNNs:

$$
\widehat{f}(x)=W^{T} S(x),
$$

where $W \in R^{q \times m}$ is the adjustable weight matrix and $q$ is the number of neurons. $x \in \Omega_{x}, \Omega_{x}$ is a compact set. $S(x)=\left[s_{1}(x), s_{2}(x), \ldots, s_{q}(x)\right]^{T}$ is a function vector, where $s_{j}(x)=\exp \left[-\left(x-\mu_{j}\right)^{T}\left(x-\mu_{j}\right) / \varphi_{j}\right], j=1,2, \ldots, q, \mu_{j}=$ $\left[\mu_{j 1}, \mu_{j 2}, \ldots, \mu_{j n}\right]^{T}$ represents the center of receptive field. $\varphi_{j}$ is the width of the Gaussian function.

For any smooth function $f(x)$, by choosing the designed parameters and making the neuron number $q$ large enough, 
RBFNNs based method can approximate the function to any desired accuracy over a compact set $\Omega$. There exists an ideal weights matrix $W^{*}$ such that

$$
f(x)=W^{*} S(x)+\varepsilon(x),
$$

where $\varepsilon(x) \in R^{m}$ is the approximation error, which satisfies $\|\varepsilon(x)\| \leq l$, and $l$ is a positive constant. The approximation error represents possible deviation between the unknown nonlinear term $f(x)$ and the ideal approximation $W^{*} S(x)$. Large practical applications have proved that we can decrease the approximation error $\varepsilon(x)$ by choosing enough neural network node $q$.

The ideal unknown weight matrix $W^{*}$ is an "artificial" quantity for the purpose of analysis, it is defined as the following form:

$$
W^{*}=\arg \min _{W \in R^{q \times m}}\left\{\sup _{x \in \Omega_{x}}\left\|f(x)-W^{T} S(x)\right\|\right\} .
$$

\section{Main Results}

This section investigates adaptive consensus of the fractionalorder nonlinear MASs with external disturbance based on the RBFNNs method, where we use the RBFNNs based method to approximate the nonlinear terms in MASs, and a valid control law is designed to achieve adaptive consensus. Firstly, to discuss the MASs stability, a Lyapunov candidate function is used, and the known nonlinear terms are assumed to obtain the useful stability results based on fractional theory. Secondly, adaptive consensus with the unknown nonlinear terms is studied based on the above results, the nonlinear terms are approximated by using the RBFNNs based method, and an effective control law is designed to achieve adaptive consensus of MASs.

3.1. Consensus with Known Nonlinear Terms. In the subsection, consensus is mainly discussed when the nonlinear terms are known under the undirected connected graph $G$. A valid control law is given, and some stability results are obtained.

Consider the fractional-order MASs with $n$ agents; their systems are shown as the following form:

$$
x_{i}^{(\alpha)}(t)=f_{i}\left(x_{i}(t)\right)+u_{i}(t)+d_{i}\left(x_{i}(t)\right), \quad i \in N
$$

where $x_{i}^{(\alpha)}(t)$ is a simple notation of the fractional derivative ${ }_{a}^{C} D_{t}^{\alpha} x_{i}(t), \alpha \in(0,1)$, and $N=(1,2, \ldots, n) . f_{i}\left(x_{i}(t)\right): R^{m} \longrightarrow$ $R^{m}$ is the smooth nonlinear term with uncertainty; $x_{i}(t) \in$ $R^{m}$ and $u_{i}(t) \in R^{m}$ represent the state vector and the control input vector, respectively. $d_{i}\left(x_{i}(t)\right)$ is the nonlinear external disturbance. follows.

Define the consensus error vector for the ith agent as

$$
\xi_{i}(t)=\sum_{j=1}^{n} a_{i j}\left[x_{i}(t)-x_{j}(t)\right] . \quad i, j \in N, i \neq j
$$

To study the system stability, we define the following Lyapunov candidate function:

$$
V(t)=\frac{1}{2} x^{T}(t)\left(L \otimes I_{m}\right) x(t)
$$

where $x(t)=\left(x_{1}^{T}(t), \ldots, x_{n}^{T}(t)\right)^{T} \in R^{n m}$. According to Lemma 3, we can get that $V(t)$ is a symmetric positive semidefinite function.

Because $L$ is a symmetric positive semidefinite matrix, there exist $n$ eigenvalues $\lambda_{1}, \ldots, \lambda_{n}$, and $p_{11}, \ldots, p_{1 m}$, $p_{21}, \ldots, p_{2 m} \cdots p_{n 1}, \ldots, p_{n m}$ can be chosen as the eigenvectors of matrix $L \otimes I_{m}$ corresponding to eigenvalues $\lambda_{1}, \ldots, \lambda_{n}$. Define $P=\left[p_{11}, \ldots, p_{n m}\right] \in R^{n m \times n m}$; then, $P^{T} P=P P^{T}=I_{n m}$ can be guaranteed.

Based on the above analysis, $V(t)$ can be rewritten as

$$
\begin{aligned}
V(t) & =\frac{1}{2} x^{T}(t)\left(L \otimes I_{m}\right) x(t)=\frac{1}{2} x^{T}(t) P^{T} \Lambda P x(t) \\
& =\frac{1}{2} x^{T}(t) P^{T} \sqrt{\Lambda} \sqrt{\Lambda} P x(t) \\
& =\frac{1}{2} x^{T}(t) P^{T} \sqrt{\Lambda} \sqrt{\widehat{\Lambda}} \sqrt{\widehat{\Lambda}^{-1}} \sqrt{\widehat{\Lambda}^{-1}} \sqrt{\widehat{\Lambda}} \sqrt{\Lambda} P x(t) \\
& =\frac{1}{2} x^{T}(t) P^{T} \Lambda P P^{T} \widehat{\Lambda}^{-1} P P^{T} \Lambda P x(t) \\
& =\frac{1}{2} x^{T}(t)\left(L \otimes I_{m}\right)^{T} P^{T} \widehat{\Lambda}^{-1} P\left(L \otimes I_{m}\right) x(t) \\
& =\frac{1}{2} \xi^{T}(T) P^{T} \widehat{\Lambda}^{-1} P \xi(t)=\frac{1}{2} \xi^{T}(T) \Delta \xi(t),
\end{aligned}
$$

where $\Lambda=\operatorname{diag}\left\{0, \lambda_{2} I_{m}, \ldots, \lambda_{n} I_{m}\right\}, \widehat{\Lambda}=\operatorname{diag}\left\{\lambda_{2} I_{m}, \lambda_{2} I_{m}, \ldots\right.$, $\left.\lambda_{n} I_{m}\right\}, \xi(t)=\left(\xi_{1}^{T}(t), \ldots, \xi_{n}^{T}(t)\right)$, and $\Delta=P^{T} \widehat{\Lambda}^{-1} P$. Then, the following result can be given:

$$
\frac{\lambda_{\min }(\Delta)}{2} \sum_{i=1}^{n}\left\|\xi_{i}(t)\right\|^{2} \leq V(t) \leq \frac{\lambda_{\max }(\Delta)}{2} \sum_{i=1}^{n}\left\|\xi_{i}(t)\right\|^{2}
$$

where $\lambda_{\text {min }}$ and $\lambda_{\text {max }}$ represent the smallest eigenvalue and the largest eigenvalue of matrix $\Delta$, respectively.

Taking time fractional derivative of $V(t)$, based on equality (15) and Lemma 2, we can obtain

$$
\begin{aligned}
& V^{(\alpha)}(t) \leq x^{T}(t)\left(L \otimes I_{m}\right) x^{(\alpha)}(t)=\sum_{i=1}^{n} \xi_{i}^{T}(t) x_{i}^{(\alpha)}(t) \\
& =\sum_{i=1}^{n} \xi_{i}^{T}(t)\left[f_{i}\left(x_{i}(t)\right)+u_{i}(t)+d_{i}\left(x_{i}(t)\right)\right] \\
& \quad \leq \sum_{i=1}^{n}\left[\xi_{i}^{T}(t) f_{i}\left(x_{i}(t)\right)+\xi_{i}^{T}(t) u_{i}(t)\right. \\
& \left.+\xi_{i}^{T}(t) d_{i}\left(x_{i}(t)\right)\right] .
\end{aligned}
$$


Because $a b \leq a^{2} / 2+b^{2} / 2$, we have the following inequality:

$$
\xi_{i}^{T}(t) d_{i}\left(x_{i}(t)\right) \leq \frac{\beta_{i}^{2}}{2}+\frac{\left\|\xi_{i}(t)\right\|^{2} \cdot\left\|d_{i}\left(x_{i}(t)\right)\right\|^{2}}{2 \beta_{i}^{2}} .
$$

The inequality (19) can be rewritten as the following form:

$$
\begin{gathered}
V^{(\alpha)}(t) \leq \sum_{i=1}^{n}\left[\xi_{i}^{T}(t) f_{i}\left(x_{i}(t)\right)+\xi_{i}^{T}(t) u_{i}(t)\right. \\
\left.+\frac{1}{2 \beta_{i}^{2}} \xi_{i}^{T}(t) \xi_{i}(t)\left\|d_{i}\left(x_{i}(t)\right)\right\|^{2}\right]+\frac{1}{2} \sum_{i=1}^{n} \beta_{i}^{2} \\
=\sum_{i=1}^{n}\left[\xi_{i}^{T}(t) Q_{i}\left(x_{i}(t)\right)+\xi_{i}^{T}(t) u_{i}(t)\right]+\frac{1}{2} \sum_{i=1}^{n} \beta_{i}^{2},
\end{gathered}
$$

where $Q_{i}\left(x_{i}\right)=f_{i}\left(x_{i}(t)\right)+\left(1 / 2 \beta_{i}^{2}\right) \xi_{i}(t)\left\|d_{i}\left(x_{i}(t)\right)\right\|^{2}$, where $x_{i}=$ $\left\{x_{i}(t), \xi_{i}(t)\right\} \in \Omega_{x_{i}}, \Omega_{x_{i}}$ is a compact set.

The nonlinear terms in MASs (14) include $f_{i}\left(x_{i}(t)\right)$ and external disturbance $d_{i}\left(x_{i}(t)\right)$; if the nonlinear terms are known, we can design the following control law to achieve adaptive consensus:

$$
u_{i}(t)= \begin{cases}-k_{i} \xi_{i}(t)-Q_{i}\left(x_{i}(t)\right), & \xi_{i}(t) \in \Omega_{\nu_{i}}^{0} \\ 0, & \xi_{i}(t) \in \Omega_{v_{i}}\end{cases}
$$

where $k_{i} \in R^{+}, \Omega_{v_{i}} \subset \Omega_{x_{i}}$,

$$
\begin{aligned}
& \Omega_{v_{i}}=\left\{\xi_{i}(t) \mid\left\|\xi_{i}(t)\right\|<\nu_{i}\right\}, \\
& \Omega_{v_{i}}^{0}=\Omega_{x_{i}}-\Omega_{v_{i}}, \quad i \in N
\end{aligned}
$$

where $v_{i}$ is an arbitrary small constant and $\Omega_{\nu_{i}}^{0}$ is also a compact set [38].

If $\xi_{i}(t) \in \Omega_{\nu_{i}}^{0}$, the following inequality can be given based on inequality (21) and control law (22):

$$
\begin{aligned}
V^{(\alpha)}(t) \leq & \sum_{i=1}^{n}\left[\xi_{i}^{T}(t) Q_{i}\left(x_{i}(t)\right)+\xi_{i}^{T}(t) u_{i}(t)\right] \\
& +\frac{1}{2} \sum_{i=1}^{n} \beta_{i}^{2}=-\sum_{i=1}^{n} k_{i} \xi_{i}^{T}(t) \xi_{i}(t)+\frac{1}{2} \sum_{i=1}^{n} \beta_{i}^{2} \\
= & -\sum_{i=1}^{n} k_{i}\left\|\xi_{i}(t)\right\|^{2}+\frac{1}{2} \sum_{i=1}^{n} \beta_{i}^{2} .
\end{aligned}
$$

Let

$$
k_{i}=\frac{r_{i} \lambda_{\max }(\Delta)}{2}
$$

where $r_{i}$ is positive constant; then

$$
\begin{aligned}
V^{(\alpha)}(t) & \leq-\sum_{i=1}^{n} \frac{r_{i} \lambda_{\max }(\Delta)}{2}\left\|\xi_{i}(t)\right\|^{2}+\frac{1}{2} \sum_{i=1}^{n} \beta_{i}^{2} \\
& \leq-\frac{r}{2} \lambda_{\max }(\Delta) \sum_{i=1}^{n}\left\|\xi_{i}(t)\right\|^{2}+\eta \\
& \leq-r V(t)+\eta,
\end{aligned}
$$

where $r=\min \left\{r_{1}, r_{2}, \ldots, r_{n}\right\}, \eta=(1 / 2) \sum_{i=1}^{n} \beta_{i}^{2}$.

Let $m(t)=V(t)-\eta / r$; inequality (26) changes into

$$
{ }_{a}^{C} D_{t}^{\alpha} m(t) \leq-r m(t),
$$

and, then, we can find a nonnegative function $n(t)$ and get the following equality

$$
{ }_{a}^{C} D_{t}^{\alpha} m(t)+n(t)=-r m(t) .
$$

Denote $M(s)=L(m(t)), N(s)=L(n(t))$ and study (29) and its Laplace transform; then

$$
s^{\alpha} M(s)-m(0) s^{\alpha-1}+N(s)=-r M(s) .
$$

And

$$
M(s)=\frac{m(0) s^{\alpha-1}-N(s)}{s^{\alpha}+r M(s)} .
$$

we can obtain the unique solution of (29). Based on the inverse Laplace transform, $m(t)$ can be obtained as

$$
m(t)=m(0) E_{\alpha}\left(-r t^{\alpha}\right)-n(t) *\left[t^{\alpha-1} E_{\alpha, \alpha}\left(-r t^{\alpha}\right)\right] .
$$

$t^{\alpha-1}$ and $E_{\alpha, \alpha}\left(-r t^{\alpha}\right)$ are nonnegative; then, we can get the following relationship based on the above result:

$$
V(t)-\frac{\eta}{r}=m(t) \leq m(0) E_{\alpha}\left(-r t^{\alpha}\right) \longrightarrow 0 .
$$

Because $V(t) \geq\left(\lambda_{\min }(\Delta) / 2\right) \sum_{i=1}^{n}\left\|\xi_{i}(t)\right\|^{2}$,

$$
\lim _{t \rightarrow \infty} \sum_{i=1}^{n}\left\|\xi_{i}(t)\right\|^{2} \leq \frac{2 \eta}{r \lambda_{\min }(\Delta)} .
$$

Based on the definition of $\xi_{i}(t)$, there exists a constant $\varsigma$ and $T>0$; if $t>T$, then,

$$
\lim _{t \longrightarrow \infty}\left\|\xi_{i}(t)\right\| \leq \varsigma, \quad i \in N .
$$

Here $\varsigma$ is a constant, which depends on the external disturbance. We can conclude that $x_{1}, x_{2}, \ldots, x_{n}$ converge to a small constant region, and the consensus can be achieved when $\xi_{i} \in \Omega_{\nu_{i}}^{0}$. 
3.2. Adaptive Consensus with Unknown Nonlinear Terms. We can see that control law (22) cannot be used directly; because functions $f_{i}\left(x_{i}(t)\right)$ and $d_{i}\left(x_{i}(t)\right)$ are unknown, so function $Q_{i}\left(x_{i}(t)\right)$ is unknown. To solve the above problem, the RBFNNs based method is applied to estimate the unknown $Q_{i}\left(x_{i}(t)\right)$ as follows:

$$
Q_{i}\left(x_{i}(t)\right)=W_{i}^{*} S_{i}\left(x_{i}(t)\right)+\varepsilon_{i}\left(x_{i}(t)\right)
$$

where $W_{i}^{*} \in R^{m \times q_{i}}$ denotes the ideal NNs weight matrix, $q_{i}$ represents the number of neurons, $S_{i}\left(x_{i}\right) \in R^{q_{i}}$ is defined as basis function, and $\varepsilon_{i} \in R^{m}$ describes approximation error, which satisfies $\left\|\varepsilon_{i}\right\| \leq \rho_{i}$, where $\rho_{i}$ is a positive constant.

By using above RBFNNs approximation form, we design the following control law for the nonlinear MASs (14):

$$
\begin{aligned}
& u_{i}(t) \\
& = \begin{cases}-k_{i} \xi_{i}(t)-\theta_{i} \widehat{w}_{i}(t)\left\|S_{i}\left(x_{i}\right)\right\|^{2} \xi_{i}(t), & \xi_{i}(t) \in \Omega_{v_{i}}^{0} \\
0, & \xi_{i}(t) \in \Omega_{v_{i}}\end{cases}
\end{aligned}
$$

where $\widehat{w}_{i}(t)$ is used to estimate the unknown constant $w_{i}^{*}$, $w_{i}^{*}=\left\|W_{i}^{*}\right\|_{F}^{2}$.

The following equality designs the fractional-order adaption law:

$$
\widehat{w}_{i}^{(\alpha)}(t)=\delta_{i}\left(\theta_{i}\left\|S_{i}\left(x_{i}\right)\right\|^{2}\left\|\xi_{i}(t)\right\|^{2}-\sigma_{i} \widehat{w}_{i}\right),
$$

where $\delta_{i}, \theta_{i}, \sigma_{i}$ are the designed positive constants.

Remark 4. Compared to the tracking problem based on the NNs weight estimation matrix in [35], the designed adaption law uses the information of matrix norm, which will decrease the systems computational burden greatly.

Theorem 5. For the fractional-order nonlinear MASs (14) with external disturbance, if the undirected communication graph is connected, control law (37) solves the adaptive consensus problem with the consensus error among agents remaining in a small neighborhood of the origin, and

$$
k_{i}=r_{i}\left(\frac{\lambda_{\max }(\Delta)}{2}+\frac{1}{r_{i}}\right)
$$

where $r_{i}$ is positive constant and $\Delta$ is defined as equality (17).

Proof. Consider Lyapunov function as follows:

$$
V(t)=\frac{1}{2} x^{T}(t)\left(L \otimes I_{m}\right) x(t)+\frac{1}{2} \sum_{i=1}^{n} \delta_{i}^{-1} \widetilde{w}_{i}^{2}(t),
$$

where $\widetilde{w}_{i}(t)=\widehat{w}_{i}(t)-w_{i}^{*}(t)$; we can be obtained the following inequality

$$
\begin{aligned}
V^{(\alpha)}(t) \leq & \sum_{i=1}^{n}\left[\xi_{i}^{T}(t) u_{i}(t)+\xi_{i}^{T}(t) Q_{i}\left(x_{i}\right)\right] \\
& +\sum_{i=1}^{n} \delta_{i}^{-1} \widetilde{w}_{i}(t) \widetilde{w}_{i}^{(\alpha)}(t)+\frac{1}{2} \sum_{i=1}^{n} \beta_{i}^{2},
\end{aligned}
$$

and, then, subsisting equality (36) into (41), we have

$$
\begin{aligned}
& V^{(\alpha)}(t) \\
& \leq \sum_{i=1}^{n}\left[\xi_{i}^{T}(t) u_{i}(t)+\xi_{i}^{T}(t)\left(W_{i}^{*} S_{i}\left(z_{i}\right)+\varepsilon_{i}\left(z_{i}\right)\right)\right] \\
& \quad+\sum_{i=1}^{n} \delta_{i}^{-1} \widetilde{w}_{i}(t) \widetilde{w}_{i}^{(\alpha)}(t)+\frac{1}{2} \sum_{i=1}^{n} \beta_{i}^{2},
\end{aligned}
$$

due to

$$
\begin{aligned}
\xi_{i}^{T}(t) W_{i}^{*} S_{i}\left(z_{i}\right) & \leq \theta_{i}\left\|\xi_{i}(t)\right\|^{2}\left\|W_{i}^{*} S_{i}\left(z_{i}\right)\right\|^{2}+\frac{1}{4 \theta_{i}} \\
& \leq \theta_{i} w_{i}^{*}\left\|\xi_{i}(t)\right\|^{2}\left\|S_{i}\left(z_{i}\right)\right\|^{2}+\frac{1}{4 \theta_{i}} \\
\xi_{i}^{T}(t) \varepsilon\left(z_{i}\right) & \leq\left\|\xi_{i}(t)\right\|^{2}+\frac{\left\|\mathcal{E}\left(z_{i}\right)\right\|^{2}}{4} \\
& \leq\left\|\xi_{i}(t)\right\|^{2}+\frac{\left\|\rho_{i}\right\|^{2}}{4} .
\end{aligned}
$$

Using control law (37), inequality (42) can be rewritten as

$$
\begin{aligned}
& V^{(\alpha)}(t) \leq \sum_{i=1}^{n}\left[\xi_{i}^{T}(t) u_{i}(t)+\theta_{i} w_{i}^{*}\left\|\xi_{i}(t)\right\|^{2}\left\|S_{i}\left(z_{i}\right)\right\|^{2}\right. \\
& \left.+\left\|\xi_{i}(t)\right\|^{2}\right]+\sum_{i=1}^{n} \delta_{i}^{-1} \widetilde{w}_{i}(t) \widetilde{w}_{i}^{(\alpha)}(t)+\sum_{i=1}^{n}\left(\frac{1}{2} \beta_{i}^{2}+\frac{1}{4 \theta_{i}}\right. \\
& \left.+\frac{\rho_{i}^{2}}{4}\right) \leq \sum_{i=1}^{n}\left[-k_{i} \xi_{i}^{T}(t) \xi_{i}(t)\right. \\
& -\theta_{i} \widehat{w}_{i}\left\|S_{i}\left(z_{i}\right)\right\|^{2} \xi_{i}^{T}(t) \xi_{i}(t) \\
& \left.+\theta_{i} w_{i}^{*}\left\|\xi_{i}(t)\right\|^{2}\left\|S_{i}\left(z_{i}\right)\right\|^{2}+\left\|\xi_{i}(t)\right\|^{2}\right]+\sum_{i=1}^{n} \widetilde{w}_{i}(t) \\
& +\left(\theta_{i}\left\|\xi_{i}(t)\right\|^{2}\left\|S_{i}\left(z_{i}\right)\right\|^{2}-\sigma_{i} \widehat{w}_{i}(t)\right)+\sum_{i=1}^{n}\left(\frac{1}{2} \beta_{i}^{2}\right. \\
& \left.+\frac{1}{4 \theta_{i}}+\frac{\rho_{i}^{2}}{4}\right) \leq \sum_{i=1}^{n}\left(-k_{i} \xi_{i}^{T}(t) \xi_{i}(t)+\left\|\xi_{i}(t)\right\|^{2}\right. \\
& +\theta_{i} \widehat{w}_{i}\left\|\xi_{i}(t)\right\|^{2}\left\|S_{i}\left(z_{i}\right)\right\|^{2} \\
& \left.+\theta_{i} w_{i}^{*}\left\|\xi_{i}(t)\right\|^{2}\left\|S_{i}\left(z_{i}\right)\right\|^{2}\right)+\sum_{i=1}^{n} \widetilde{w}_{i}(t)
\end{aligned}
$$




$$
\begin{aligned}
& \cdot\left(\theta_{i}\left\|\xi_{i}(t)\right\|^{2}\left\|S_{i}\left(z_{i}\right)\right\|^{2}-\sigma_{i} \widehat{w}_{i}(t)\right)+\sum_{i=1}^{n}\left(\frac{1}{2} \beta_{i}^{2}\right. \\
& \left.+\frac{1}{4 \theta_{i}}+\frac{\rho_{i}^{2}}{4}\right) \leq-\sum_{i=1}^{n}\left[\left(k_{i}-1\right)\left\|\xi_{i}(t)\right\|^{2}\right] \\
& -\sum_{i=1}^{n} \sigma_{i} \widetilde{w}_{i}(t) \widehat{w}_{i}(t)+\sum_{i=1}^{n}\left(\frac{1}{2} \beta_{i}^{2}+\frac{1}{4 \theta_{i}}+\frac{\rho_{i}^{2}}{4}\right),
\end{aligned}
$$

because

$$
\widetilde{w}_{i}(t) \widehat{w}_{i}(t)=\frac{1}{2} \widetilde{w}_{i}^{2}(t)+\frac{1}{2} \widehat{w}_{i}^{2}(t)-\frac{1}{2} w_{i}^{* 2},
$$

so that

$$
-\sigma_{i} \widetilde{w}_{i}(t) \widehat{w}_{i}(t) \leq-\frac{1}{2} \sigma_{i} \widetilde{w}_{i}^{2}(t)+\frac{1}{2} \sigma_{i} w_{i}^{* 2},
$$

and, then, we can obtain the following result from inequality (45)

$$
\begin{aligned}
V^{(\alpha)}(t) \leq & -\sum_{i=1}^{n} \frac{r_{i}}{2} \lambda_{\max }(\Delta)\left\|\xi_{i}(t)\right\|^{2}-\frac{1}{2} \sum_{i=1}^{n} \sigma_{i} \widetilde{w}_{i}(t) \\
& +\sum_{i=1}^{n}\left(\frac{1}{2} \beta_{i}^{2}+\frac{1}{4 \theta_{i}}+\frac{\rho_{i}^{2}}{4}+\frac{\sigma_{i} w^{* 2}}{2}\right) \\
\leq & -r V(t)+\eta^{\prime},
\end{aligned}
$$

where $r=\min \left(r_{1}, \ldots, r_{n}, \sigma_{1} \delta_{1}, \ldots, \sigma_{n} \delta_{n}\right) \cdot \eta^{\prime}=\sum_{i=1}^{n}\left((1 / 2) \beta_{i}^{2}+\right.$ $\left.1 / 4 \theta_{i}+\rho_{i}^{2} / 4+\sigma_{i} w^{* 2} / 2\right)$. According to the proof process in Section 3.1, we can prove that the adaptive consensus can be achieved under control law (37), and the consensus error among agents can converge in a small neighborhood of the origin.

Remark 6. The constant $\eta^{\prime}$ decides the quality of the state convergence performance, which depends on external disturbance, the RBFNNs approximation error $\varepsilon_{i}$, and the parameters $\theta_{i}, w_{i}^{*}$; the convergence performance can be improved as good as desired through changing above influencing factors.

Remark 7. Different from the results in [27-31], where the Lipschitz condition and the boundedness condition are demanded to achieve consensus of fractional-order MASs, the results in this paper do not include above rigorous conditions, and they provide a control method to solve the consensus problem for the general nonlinear fractional-order MASs.

\section{Simulations}

To prove the effectiveness of the obtained results, we consider two MASs in two-dimensional space: one contains six agents, and the other contains five agents. The predictor-corrector method for fractional-order systems is mainly used [39], where the estimation value $w^{*}$ plays a key role, which can be solved by the fractional-order adaption law.

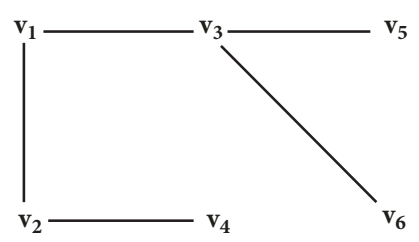

FIgURE 1: Communication graph with six agents.

In the simulations, 36 nodes are designed in RBFNNs, and $\mu_{i}$ evenly distribute in range $[-6,6] \times[-6,6]$, the width $\varphi_{j}=2 . S_{i}\left(x_{i}(t)\right)=\left[s_{1}\left(x_{i}(t), \ldots, s_{36}\left(x_{i}(t)\right)\right]^{T}\right.$, where $s_{j}\left(x_{i}(t)\right)=$ $\exp \left[-\left(x_{i}(t)-\mu_{j}(t)\right)^{T}\left(x_{i}(t)-\mu_{j}(t)\right) / \varphi_{j}^{2}\right], j=1,2, \ldots, 36$.

Firstly, we consider the communication topology graph with six agents as in Figure 1, which are connected. Choose $\alpha=0.98$; we denote the state of the $i$ th agent as $x_{i}(t)=$ $\left(x_{i 1}(t), x_{i 2}(t)\right)^{T}$; for the six agents, their initial values are given as $\left(x_{11}(0), x_{12}(0)\right)^{T}=(5,-3)^{T},\left(x_{21}(0), x_{22}(0)\right)^{T}=(10,3)^{T}$, $\left(x_{31}(0), x_{32}(0)\right)^{T}=(-3,2)^{T},\left(x_{41}(0), x_{42}(0)\right)^{T}=(3,-3)^{T}$, $\left(x_{51}(0), x_{52}(0)\right)^{T}=(-5,0)^{T},\left(x_{61}(0), x_{62}(0)\right)^{T}=(2,6)^{T}$. Let the initial value $\widehat{w}_{i}(0)=0, i=1,2, \ldots, 6$ for the fractionalorder adaption law. The systems with nonlinear term and external disturbance can be written as the following form:

$$
\begin{aligned}
{\left[\begin{array}{c}
x_{i 1}^{(\alpha)}\left(x_{i}(t)\right) \\
x_{i 2}^{(\alpha)}\left(x_{i}(t)\right)
\end{array}\right]=} & {\left[\begin{array}{l}
f_{i 1}\left(x_{i}(t)\right) \\
f_{i 2}\left(x_{i}(t)\right)
\end{array}\right]+\left[\begin{array}{l}
d_{i 1}\left(x_{i}(t)\right) \\
d_{i 2}\left(x_{i}(t)\right)
\end{array}\right] } \\
& +\left[\begin{array}{l}
u_{i 1}\left(x_{i}(t)\right) \\
u_{i 2}\left(x_{i}(t)\right)
\end{array}\right],
\end{aligned}
$$

where $f_{i 1}\left(x_{i}(t)\right)=x_{i 2}(t) \sin \left(x_{i 1}(t) / 15\right), f_{i 2}\left(x_{i}(t)\right)=$ $x_{i 1}(t) \sin \left(x_{i 2}(t) / 15\right), d_{i 1}\left(x_{i}(t)\right)=x_{i 1}(t)^{2} \cos \left(x_{i 1}(t) / 3\right)$, $d_{i 2}\left(x_{i}(t)\right)=x_{i 2}(t)^{2} \cos \left(x_{i 2}(t) / 3\right)$ are defined, and $\delta_{i}=0.4>0$, $\theta_{i}=2>0, \sigma_{i}=0.2>0, r_{i}=1 / 6>0$. Based on the Laplace matrix, we can calculate that $\lambda_{\max }(\Delta)=3.0779$. The conditions in Theorem 5 are satisfied. Figures 2(a) and 2(b) describe the agents states $x_{i 1}(t)$ and $x_{i 2}(t)$ along $\mathrm{x}$-axis and $y$-axis, respectively. From the figures, we can see that the consensus errors remain in a small neighborhood of the origin, the consensus can be achieved by using the control law (37) and the fractional-order adaption law (38), and Theorem 5 can be guaranteed. Figure 3 gives the state graph in two-dimensional space when time is changing; it also proves the obtained results.

Secondly, the communication topology graph with five agents is considered as in Figure 4, which are also connected. To verify Theorem 5 , we choose $\alpha=0.94$; the initial values are designed as $\left(x_{11}(0), x_{12}(0)\right)^{T}=(0.5,-3)^{T}$, $\left(x_{21}(0), x_{22}(0)\right)^{T}=(-2,1)^{T},\left(x_{31}(0), x_{32}(0)\right)^{T}=(3.4,-5)^{T}$, $\left(x_{41}(0), x_{42}(0)\right)^{T}=(5.8,-10)^{T},\left(x_{51}(0), x_{52}(0)\right)^{T}=(-6,6)^{T}$. The initial value $\widehat{w}_{i}(0)=0, i=1,2, \ldots, 6$, is assumed for the fractional-order adaption law. The nonlinear term and external disturbance are defined as $f_{i 1}\left(x_{i}(t)\right)=x_{i 2}^{2}(t)$, $f_{i 2}\left(x_{i}(t)\right)=x_{i 1}^{2}(t), d_{i 1}\left(x_{i}(t)\right)=2 x_{i 2}(t)^{2} \sin \left(x_{i 1}^{2}(t)\right)$, $d_{i 2}\left(x_{i}(t)\right)=2 x_{i 1}(t)^{2} \cos \left(x_{i 2}^{2}(t)\right), \delta_{i}=4>0, \theta_{i}=6.6>0$, $\sigma_{i}=1.2>0, r_{i}=3>0$. Based on the Laplace matrix, 


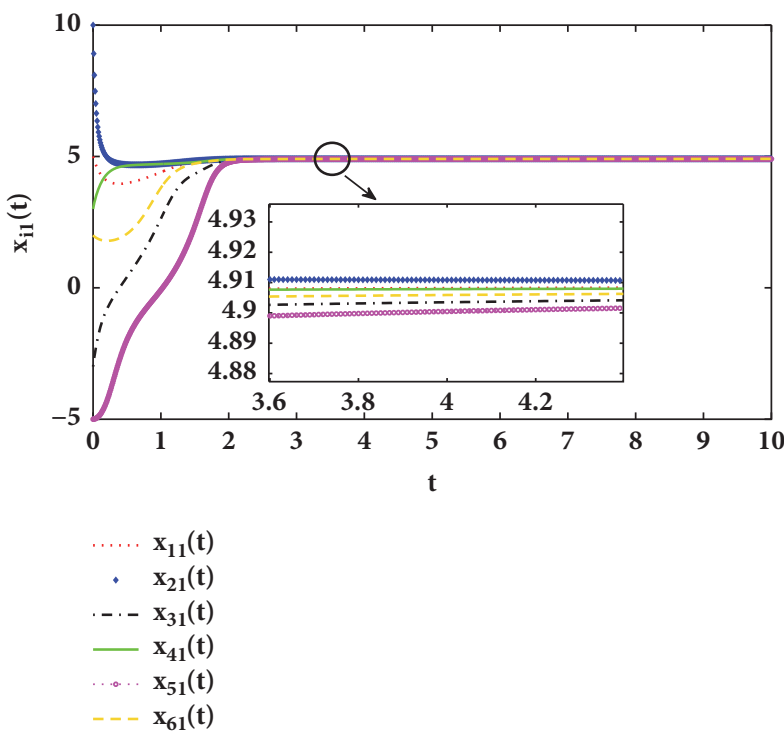

(a)

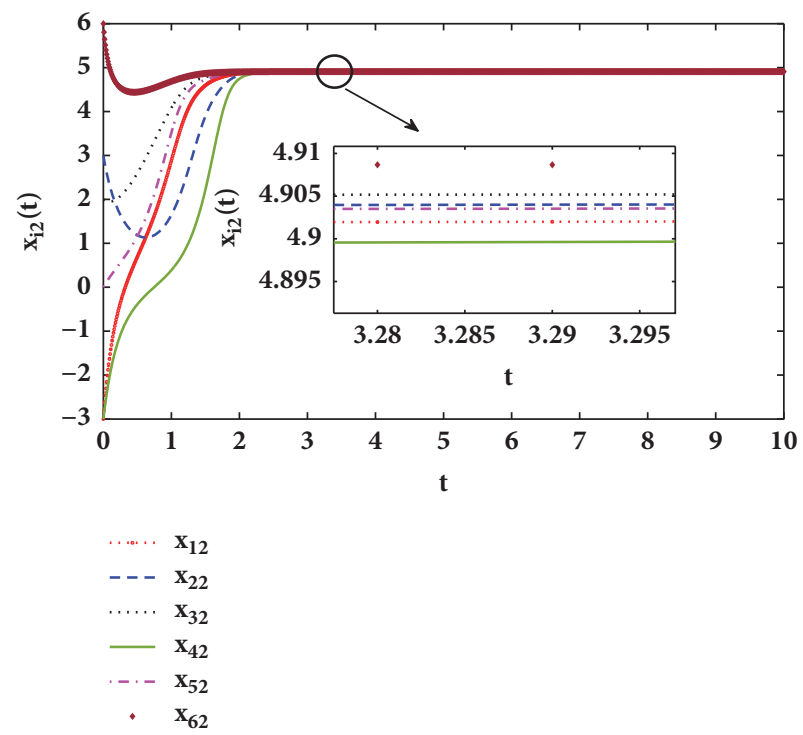

(b)

FIGURE 2: The states of the $i$ th agent $(i=1,2,3,4,5,6)$ : (a) the state $x_{i 1}$ along $x$-axis; (b) the states $x_{i 2}$ along $y$-axis.

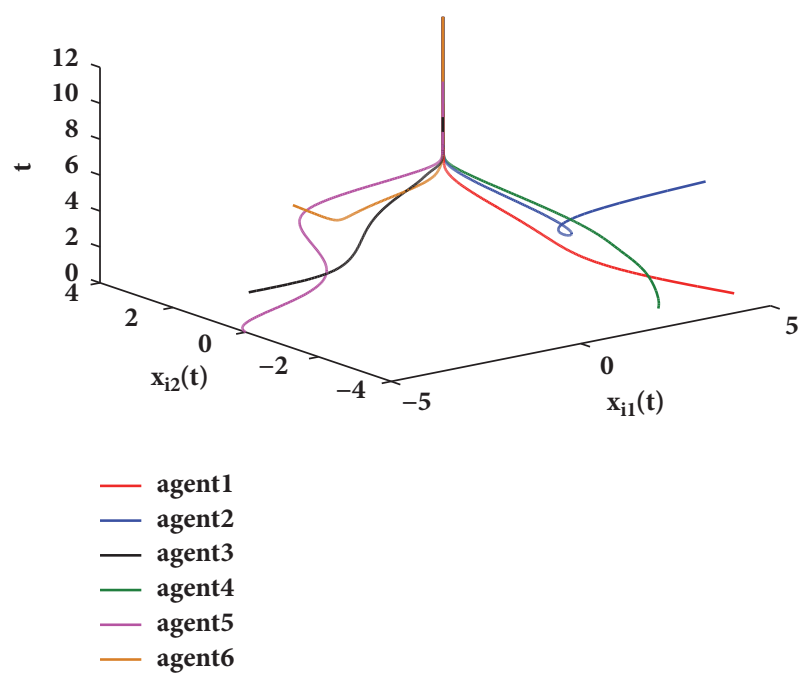

FIGURE 3: The states of six agents in two-dimensional space.

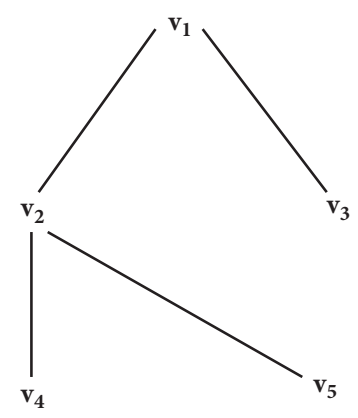

FIGURE 4: Communication graph with five agents. we can calculate that $\lambda_{\max }(\Delta)=1.9275$. The conditions for the fractional-order adaption law and Theorem 5 are satisfied. Figures 5(a) and 5(b) show the consensus errors remaining in a small neighborhood of the origin along $\mathrm{x}$-axis and $\mathrm{y}$ axis, respectively. From the figures, we can judge that the consensus can be achieved based on the control law (37) and the fractional-order adaption law (38), and the effectiveness of Theorem 5 can be proved. To show the states relationship, Figure 6 gives the state graph in two-dimensional space with time changing; it also shows that the consensus can be achieved by using the control law based on RBFNNs.

\section{Conclusion}

This paper has investigated adaptive consensus of the fractional nonlinear MASs based on the developed RBFNNs approximation control method. Firstly, the fractional derivative theories and corresponding graph theory have been given; moreover, RBFNNs based method has been explained to achieve the nonlinear terms approximation. Secondly, by using matrix theory and fractional-order theory, the valid control law and relative results have been obtained based on the known nonlinear terms, and then, to overcome the unknown nonlinear terms, the control law and a fractionalorder adaption law have been designed based on the RBFNNs method; at last, a theorem has been given to achieve adaptive consensus when the nonlinear terms are unknown, and the rigorous proof has been given to guarantee the correctness of the gotten theorem. Finally, two simulations with six agents and five agents have been discussed by using the designed control law for the uncertain nonlinear MASs, which have proved the validity of the obtained theory. Time delay is inevitable in real application, and it can cause poor 


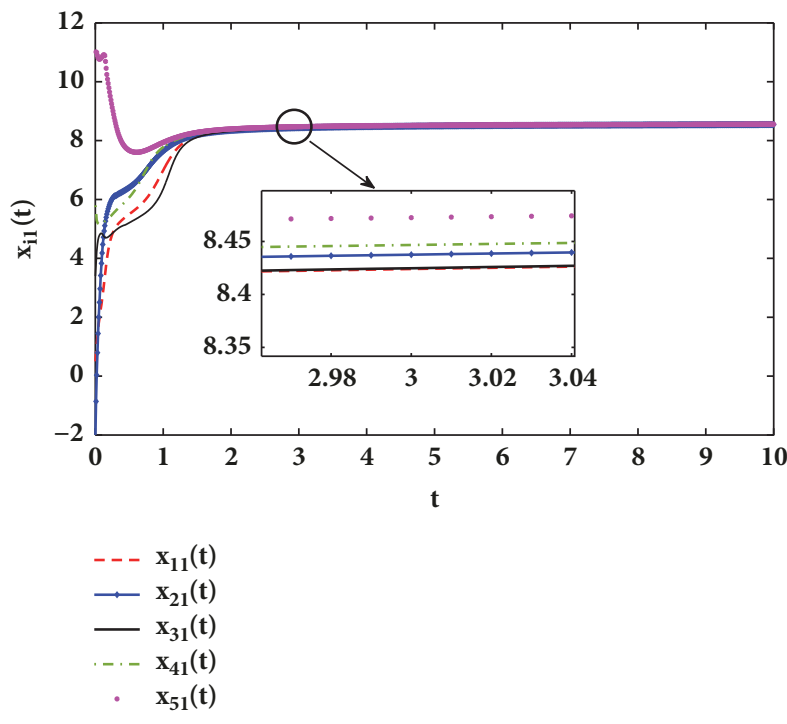

(a)

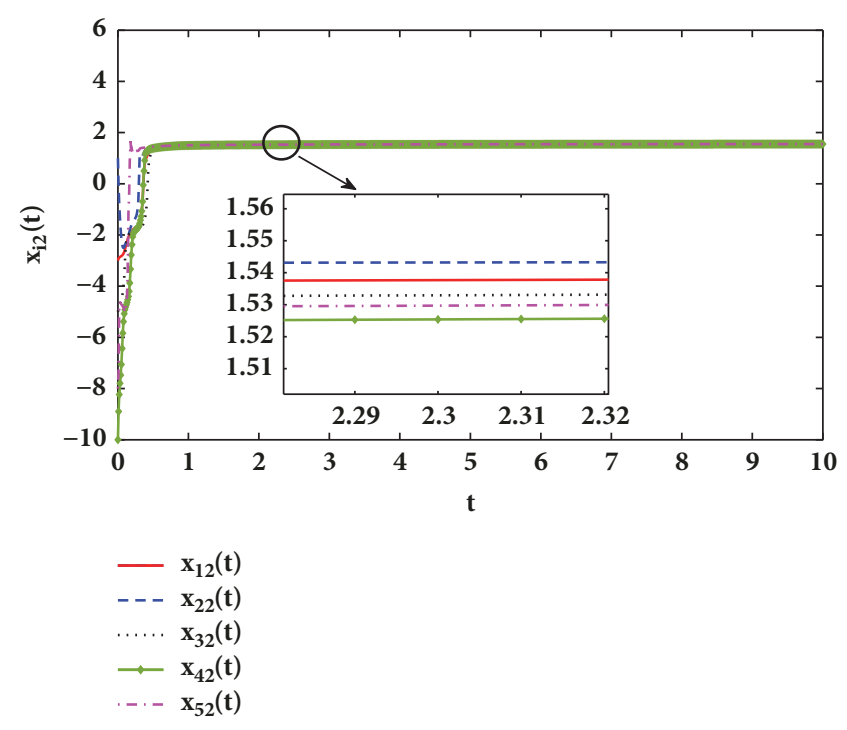

(b)

FIgURE 5: The states of the $i$ th agent $(i=1,2,3,4,5)$ : (a) the state $x_{i 1}$ along $x$-axis; (b) the states $x_{i 2}$ along $y$-axis.

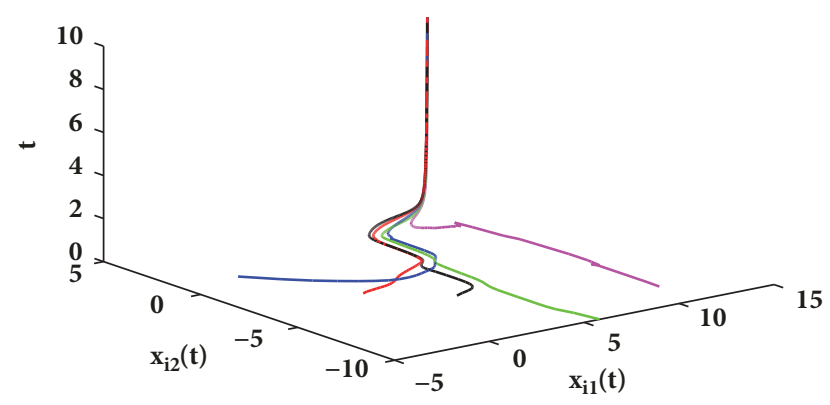

Figure 6: The states of five agents in two-dimensional space.

performance for the system stability; therefore, the study on the time delay of the fractional-order uncertain MASs will be our main work.

\section{Data Availability}

All data have been included in the paper; no additional data are available for the paper.

\section{Conflicts of Interest}

The authors declare that they have no conflicts of interest.

\section{Acknowledgments}

This work was supported by National Natural Science Foundation of China [Grant number 61703035], China Postdoctoral Science Foundation [Grant number 2017M610772], Fundamental Research Funds for the Central Universities [Grant number FRF-TP-16-011A1], and National Key R\&D Program of China [2017YFF0207400].

\section{References}

[1] M. Wooldridge, An introduction to multiagent systems, vol. 28, Wiley \& Sons, 2 edition, 2012.

[2] H. Zhao and J. H. Park, "Dynamic output feedback consensus of continuous-time networked multiagent systems," Complexity, vol. 20, no. 5, pp. 35-42, 2015.

[3] X. Ai, J. Yu, Z. Jia, D. Yang, X. Xu, and Y. Shen, "Disturbance observer-based consensus tracking for nonlinear multiagent systems with switching topologies," International Journal of Robust and Nonlinear Control, vol. 28, no. 6, pp. 2144-2160, 2018.

[4] S. Djaidja and Q. H. Wu, "Leader-following consensus for single-integrator multi-agent systems with multiplicative noises in directed topologies," International Journal of Systems Science, 2014.

[5] L. Rong and H. Shen, "Distributed containment control of second-order multiagent systems with input delays under general protocols," Complexity, vol. 21, no. 6, pp. 112-120, 2016.

[6] B. Kaviarasan, R. Sakthivel, and S. Abbas, "Robust consensus of nonlinear multi-agent systems via reliable control with probabilistic time delay," Complexity, 2016.

[7] M. Lu and L. Liu, "Consensus of linear multi-agent systems subject to communication delays and switching networks," International Journal of Robust and Nonlinear Control, vol. 27, no. 9, pp. 1379-1396, 2017.

[8] G. Wen, P. Wang, T. Huang, W. Yu, and J. Sun, "Robust NeuroAdaptive Containment of Multileader Multiagent Systems With Uncertain Dynamics," IEEE Transactions on Systems, Man, and Cybernetics: Systems, 2017.

[9] X. Ai, J. Yu, Z. Jia, Y. Shen, P. Ma, and D. Yang, "Adaptive robust consensus tracking for nonlinear second-order multiagent systems with heterogeneous uncertainties," International Journal of Robust and Nonlinear Control, vol. 27, no. 18, pp. 5082-5096, 2017.

[10] T. H. Lee, J. H. Park, D. H. Ji, and H. Y. Jung, "Leader-following consensus problem of heterogeneous multi-agent systems with nonlinear dynamics using fuzzy disturbance observer," Complexity, vol. 19, no. 4, pp. 20-31, 2014. 
[11] J. Yu and L. Wang, "Group consensus of multi-agent systems with directed information exchange," International Journal of Systems Science, vol. 43, no. 2, pp. 334-348, 2012.

[12] G. Wen, W. Yu, Z. Li, X. Yu, and J. Cao, "Neuro-Adaptive Consensus Tracking of Multiagent Systems with a HighDimensional Leader," IEEE Transactions on Cybernetics, vol. 47, no. 7, pp. 1730-1742, 2017.

[13] X. Liu, C. Du, H. Liu, and P. Lu, "Distributed event-triggered consensus control with fully continuous communication free for general linear multi-agent systems under directed graph," International Journal of Robust and Nonlinear Control, vol. 28, no. 1, pp. 132-143, 2018.

[14] I. Podlubny, Fractional Differential Equations, vol. 198 of Mathematics in Science and Engineering, Academic Press, San Diego, Calif, USA, 1999.

[15] Y. Cao, Y. Li, W. Ren, and Y. Q. Chen, "Distributed coordination algorithms for multiple fractional-order systems," in Proceedings of the 47th IEEE Conference on Decision and Control (CDC '08), pp. 2920-2925, Cancun, Mexico, December 2008.

[16] P. Balasubramaniam, D. Nishanthi, and L. A. Jarina Banu, "Robust guaranteed cost control for discrete-time systems via partially delay-dependent controller with linear fractional uncertainties," Complexity, vol. 21, no. S2, pp. 113-122, 2016.

[17] K. Mathiyalagan and K. Balachandran, "Finite-time stability of fractional-order stochastic singular systems with time delay and white noise," Complexity, vol. 21, no. S2, pp. 370-379, 2016.

[18] T. Ma, T. Li, and B. Cui, "Coordination of fractional-order nonlinear multi-agent systems via distributed impulsive control," International Journal of Systems Science, vol. 49, no. 1, pp. 1-14, 2018.

[19] J. Bai, G. Wen, A. Rahmani, and Y. Yu, "Consensus for the fractional-order double-integrator multi-agent systems based on the sliding mode estimator," IET Control Theory \& Applications, vol. 12, no. 5, pp. 621-628, 2018.

[20] H. Y. Yang, X. Zhu, and K. Cao, "Distributed coordination of fractional order multi-agent systems with communication delays," Fractional Calculus and Applied Analysis, vol. 17, no. 1, pp. 23-37, 2014.

[21] J. Bai, G. Wen, A. Rahmani, and Y. Yu, "Distributed formation control of fractional-order multi-agent systems with absolute damping and communication delay," International Journal of Systems Science, vol. 46, no. 13, pp. 2380-2392, 2015.

[22] Z. Yu, H. Jiang, and C. Hu, "Leader-following consensus of fractional-order multi-agent systems under fixed topology," Neurocomputing, vol. 149, pp. 613-620, 2015.

[23] Y. Cao and W. Ren, "Distributed formation control for fractional-order systems: dynamic interaction and absolute/ relative damping," Systems \& Control Letters, vol. 59, no. 3-4, pp. 233-240, 2010.

[24] J. Bai, G. Wen, A. Rahmani, and Y. Yu, "Distributed consensus tracking for the fractional-order multi-agent systems based on the sliding mode control method," Neurocomputing, vol. 235, pp. 210-216, 2017.

[25] G. Xu, M. Chi, D. He, Z. Guan, D. Zhang, and Y. Wu, "Fractional-order consensus of multi-agent systems with eventtriggered control," in Proceedings of the 2014 11th IEEE International Conference on Control \& Automation (ICCA), pp. 619624, Taichung, Taiwan, June 2014.

[26] Z. Yu, H. Jiang, C. Hu, and J. Yu, "Leader-following consensus of fractional-order multi-agent systems via adaptive pinning control," International Journal of Control, vol. 88, no. 9, pp. 17461756, 2015.
[27] G. Ren and Y. Yu, "Robust consensus for fractional nonlinear multi-agent systems with external disturbances," in Proceedings of the 2017 36th Chinese Control Conference (CCC), pp. 1140111407, Dalian, China, July 2017.

[28] H. Yang, F. Han, F. Liu, H. Liu, and M. Zhao, "Distributed Coordination of Fractional Dynamical Systems with Exogenous Disturbances," Mathematical Problems in Engineering, vol. 2014, Article ID 793903, 7 pages, 2014.

[29] P. Gong, "Distributed consensus of non-linear fractional-order multi-agent systems with directed topologies," IET Control Theory \& Applications, vol. 10, no. 18, pp. 2515-2525, 2016.

[30] F. Wang and Y. Yang, "Leader-following consensus of nonlinear fractional-order multi-agent systems via event-triggered control," International Journal of Systems Science, vol. 48, no. 3, pp. 571-577, 2017.

[31] R. Abdulghafor and S. Turaev, "Consensus of fractional nonlinear dynamics stochastic operators for multi-agent systems," Information Fusion, vol. 44, pp. 1-21, 2018.

[32] W. Zhu, W. Li, P. Zhou, and C. Yang, "Consensus of fractionalorder multi-agent systems with linear models via observer-type protocol," Neurocomputing, vol. 230, pp. 60-65, 2017.

[33] D. Wang, H. Ma, and D. Liu, "Distributed control algorithm for bipartite consensus of the nonlinear time-delayed multi-agent systems with neural networks," Neurocomputing, vol. 174, pp. 928-936, 2016.

[34] G. Wen, C. L. P. Chen, Y.-J. Liu, and Z. Liu, "Neural NetworkBased Adaptive Leader-Following Consensus Control for a Class of Nonlinear Multiagent State-Delay Systems," IEEE Transactions on Cybernetics, vol. 47, no. 8, pp. 2151-2160, 2017.

[35] P. Gong and W. Lan, "Adaptive robust tracking control for uncertain nonlinear fractional-order multi-agent systems with directed topologies," Automatica, vol. 92, pp. 92-99, 2018.

[36] L. Zhao and Y. M. Jia, "Neural network-based adaptive consensus tracking control for multi-agent systems under actuator faults," International Journal of Systems Science, vol. 47, no. 8, pp. 1931-1942, 2016.

[37] W. Ren and Y. Cao, Disributed coordination of multi-agent networks: emergent probelms, models, ans issues, Springer, New York, NY, USA, 2010.

[38] C. L. P. Chen, G.-X. Wen, Y.-J. Liu, and F.-Y. Wang, "Adaptive consensus control for a class of nonlinear multiagent time-delay systems using neural networks," IEEE Transactions on Neural Networks and Learning Systems, vol. 25, no. 6, pp. 1217-1226, 2014.

[39] K. Diethelm, N. J. Ford, and A. D. Freed, "A predictor-corrector approach for the numerical solution of fractional differential equations," Nonlinear Dynamics, vol. 29, no. 1-4, pp. 3-22, 2002. 


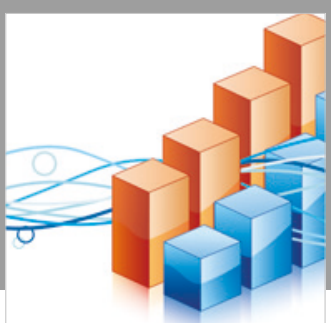

Advances in

Operations Research

\section{-n-m}
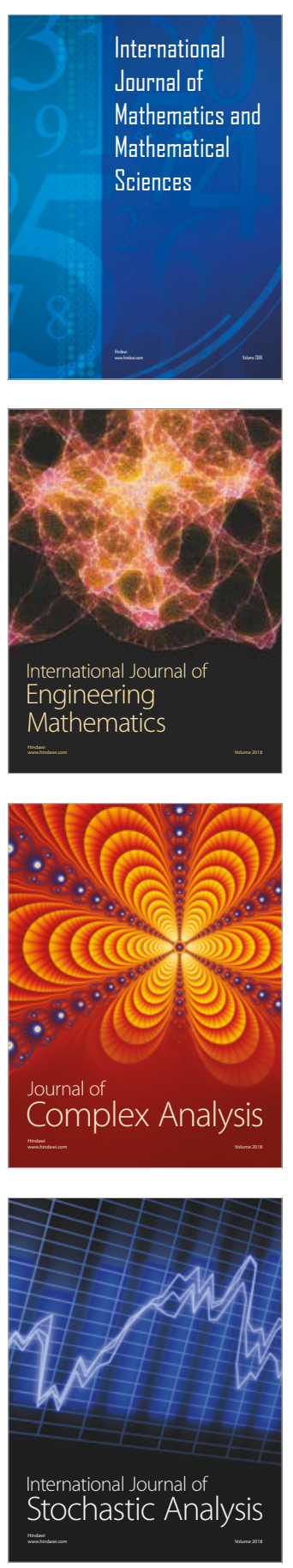
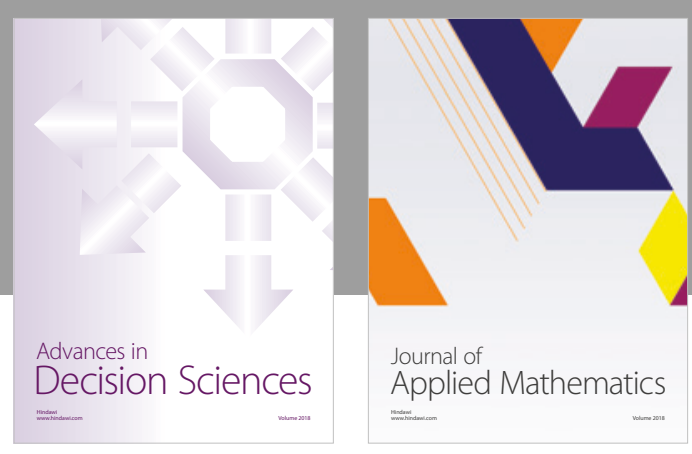

Journal of

Applied Mathematics
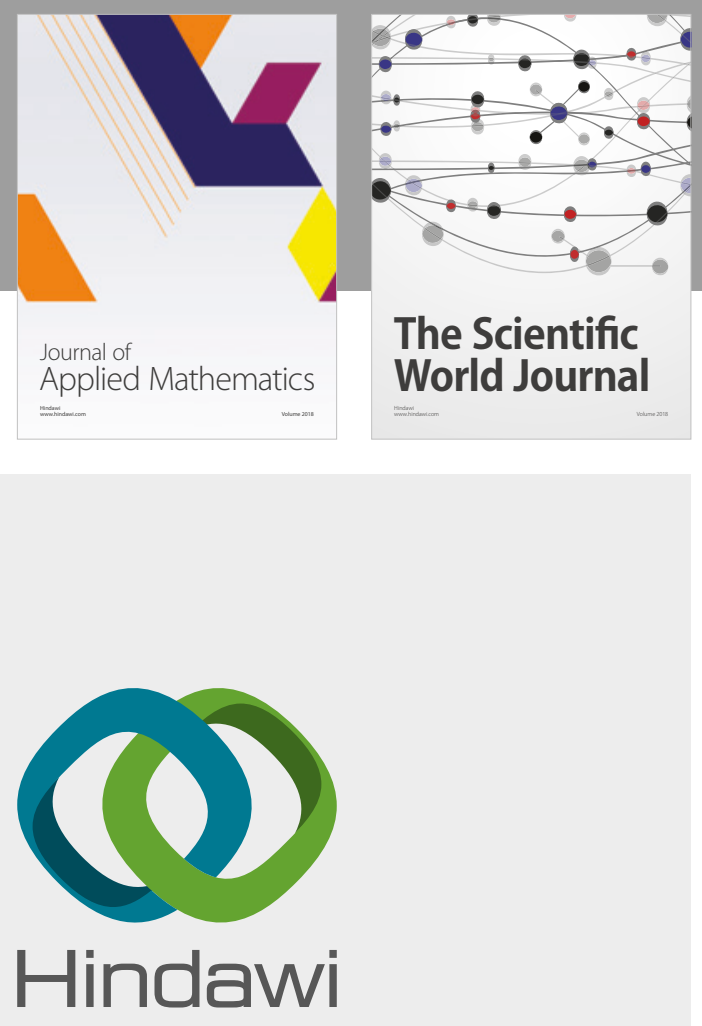

Submit your manuscripts at

www.hindawi.com

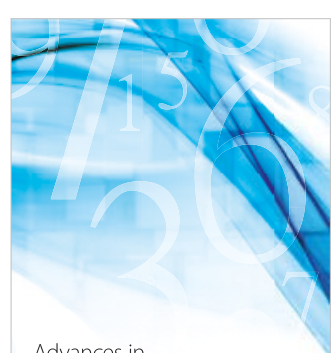

Advances in
Numerical Analysis
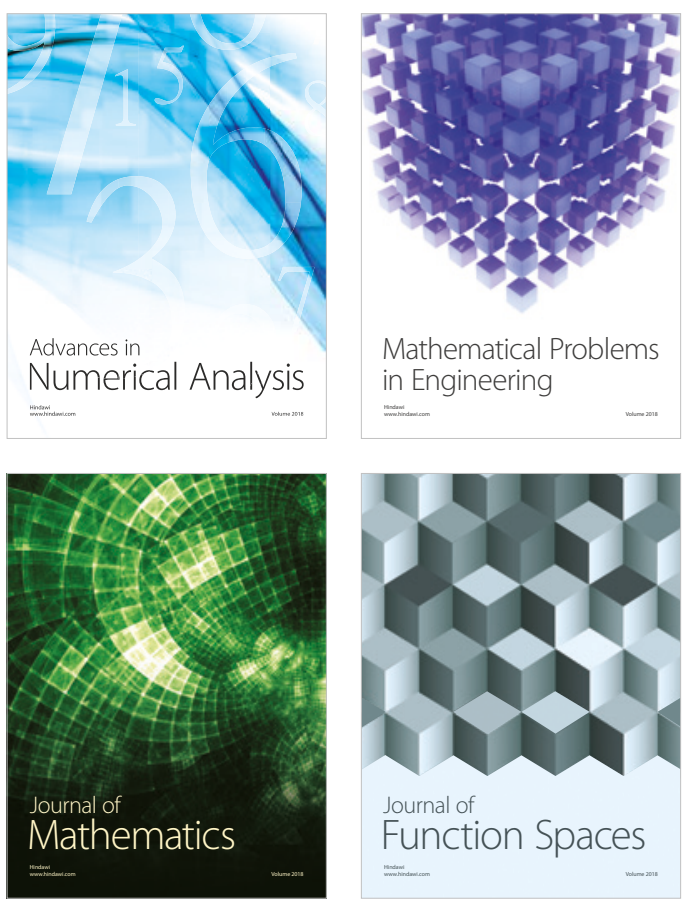

Mathematical Problems in Engineering

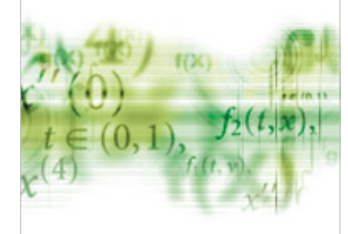

International Journal of

Differential Equations

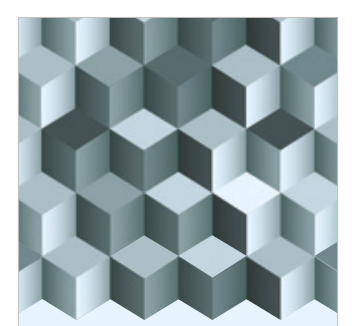

Journal of

Function Spaces
The Scientific

World Journal

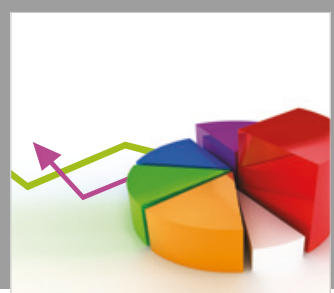

Journal of

Probability and Statistics
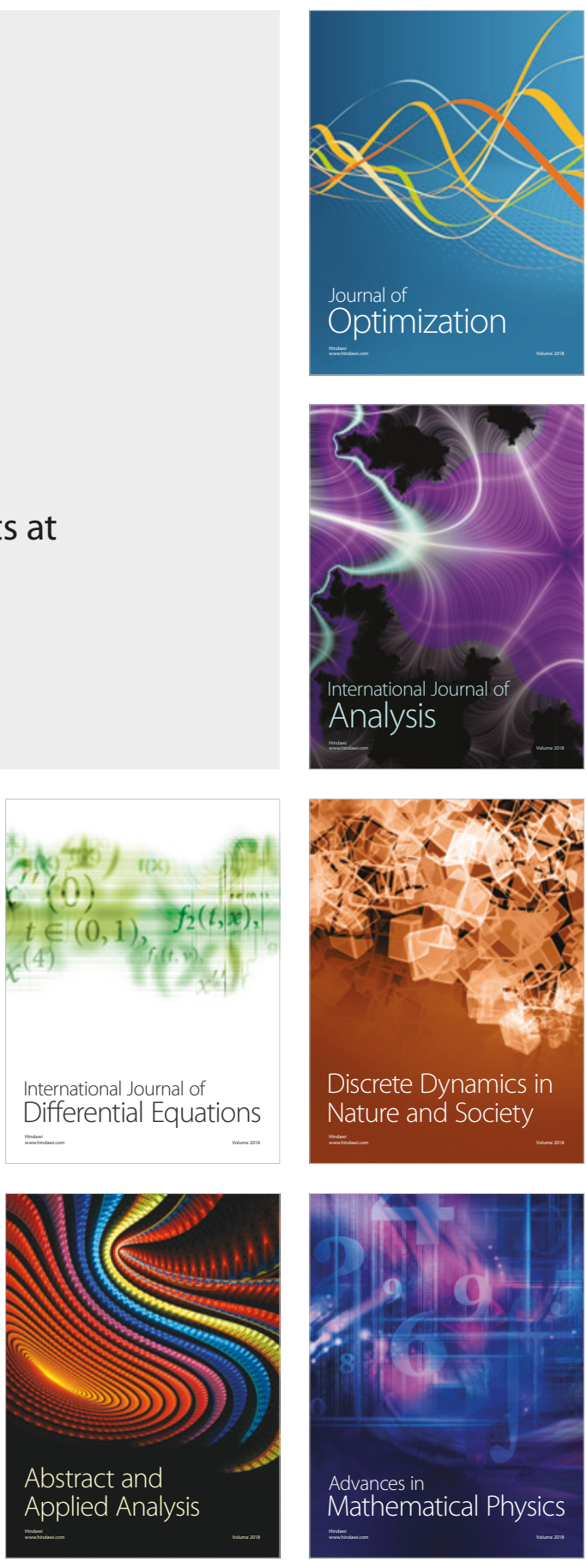